\title{
ALIMENTACIÓN ENTERAL DEL RECIÉN NACIDO PREMATURO
}

\author{
Jerónimo Zuluaga Vargas MD*, Héctor Alfonso Romero MD**
}

\section{Resumen}

En la práctica pediátrica y neonatal cada día sube la frecuencia de recién nacidos con mayor prematurez que requieren cuidados especiales dadas sus condiciones de base, con el fin de mejorar la sobrevida y disminuir las morbilidades a largo plazo. La nutrición utilizando el aparato digestivo en estos pacientes ha sido un reto importante y cambiante en el enfoque que se da a la misma, pues se ha relacionado con patologías específicas y se ha demostrado que mejorándola disminuiremos de manera importante las complicaciones futuras. Hay que tener en cuenta que la nutrición en el período neonatal tiene inferencia en el desarrollo y salud posterior del individuo en la edad adulta.

Palabras clave: alimentación enteral, prematuro, recién nacido.

Abreviaturas: RN, recién nacido (s); RNPT, recién nacido prematuro.

\section{ENTERAL NUTRITION IN THE PREMATURE INFANT}

\begin{abstract}
The frequency of newborn infants with greater prematurity who require special care, due to their basic health conditions, to improve survival and decrease long-term morbidity, is increasing every day in pediatric and neonatal practice. Nutrition using the digestive system in these patients has been a major and changing challenge as to the approach that it is given, for it has been related to specific pathologic conditions and it has been demonstrated that improving these conditions decreases future complications significantly. It must be considered that neonatal nutrition infers on the development and future health of individuals in their adult life.
\end{abstract}

Key words: enteral feeding, premature, newborn.

Fecha recibido: junio 5 de 2012 - Fecha aceptado: octubre 25 de 2012

* Residente III de Pediatría. Fundación Universitaria de Ciencias de la Salud. Bogotá DC, Colombia.
** Pediatra. Instructor Asistente. Fundación Universitaria de Ciencias de la Salud. Bogotá DC, Colombia. 


\section{Introducción}

Gracias al avance en la tecnología aplicada al cuidado de los RN se están mejorando las tasas de sobrevida de los neonatos cada vez más prematuros, pero con cierto impacto en la morbilidad a largo plazo y en el desarrollo neurológico. Si bien existen grandes avances en materia neonatal, el crecimiento posnatal de los RNPT aún está por debajo del óptimo. ${ }^{1}$ Se conøce desde hace tiempo el papel fundamental que juega la adecuada nutrición en el desarrollo de los RN pretérmino pero, a pesar de esto, los avances en el tema no han sido tan espectaculares como en otras áreas y la evidencia aún no es contundente. ${ }^{2}$ Se han recogido datos que reportan cómo la mayoría de los pacientes prematuros, en especial los menores de 30 semanas, presentan déficit nutricional (restricción de crecimiento extrauterino) al alta del hospital hasta en el 55\% de los casos, esto es multifactorial y en ocasiones iatrogénico. ${ }^{3.4}$ Este déficit nutricional puede alcanzar doce gramos de proteínas por kilo después de la primera semana de vida. ${ }^{5}$

Ya es reconocido el importante papel que juega la malnutrición en el desarrollo a largo plazo, sobretodo el neurológico.$^{6.7}$ Es así como se han realizado estudios en los que se demuestra que el perímetro cefálico en el adulto joven (importante marcador de neurodesarrollo) está relacionado con la nutrición recibida en los primeros diez días de vida. ${ }^{2.8}$ También hay estudios sobre déficit inmunológico y desarrollo de patologías neonatales propias como retinopatía del prematuro y displasia broncopulmonar, relacionados con deficiencias específicas de algunos micronutrientes (vitamina A). ${ }^{2}$ Se ha sugerido como etiología de displasia broncopulmonar el bajo aporte proteico en las primeras semanas de vida sin demostrarse una relación directa, pero continúa vigente la recomendación de dar líquidos basales bajos en los niños en riesgo. ${ }^{9}$

Teniendo en cuenta estas situaciones, el objetivo que se plantea en la actualidad es lograr un crecimiento postnatal similar al de un feto de su misma edad gestacional en el vientre materno ${ }^{10}$, pero este logro aún está un poco lejano. ${ }^{11}$ El crecimiento posnatal de los RN antes de término está disminuido respecto al crecimiento in útero, esto sumado a la pérdida fisiológica de peso que ocurre en este período y que se demora más de lo habitual para recuperarse (entre tres y cuatro semanas), pone al prematuro en una situación adversa convirtiendo la alimentación del $\mathrm{RN}$ en una prioridad y una urgencia. ${ }^{12}$ En términos generales se sugiere una ganancia de peso alrededor de $15 \mathrm{~g} / \mathrm{k} /$ día para lograr un adecuado "catch up" posnatal, ajustando los aportes nutricionales diarios con este objetivo. ${ }^{13}$ Lograrlo temprano (seis a nueve meses de vida) es de vital importancia y constituye un importante marcador de adecuado neurodesarrollo posterior. ${ }^{14}$

Se ha demostrado como los RNPT demoran más en recuperar el peso al nacer (más allá de las dos semanas de vida), como consecuencia de la falta de aporte de los nutrientes necesarios. Es por esto que algunos grupos han planteado la estrategia de intervención nutricional precoz y agresiva con el fin de mejorar el crecimiento. Esta sugerencia en esencia plantea un aporte mediante alimentación parenteral desde el primer día de vida con proteínas, lípidos desde el segundo y nutrición enteral trófica también en las primeras 24 horas, con lo que se logra un mejor crecimiento en el período neonatal sin aumento de las complicaciones ${ }^{15-18}$, incluso en algunas publicaciones recomiendan el inicio pleno de proteínas y de lípidos desde las primeras horas de vida. ${ }^{19.20}$ Existen temores sobre los posibles riesgos de estas estrategias en especial por el aumento de enterocolitis necrotizante y la toxicidad por la nutrición parenteral, y más reciente la relación entre el crecimiento rápido y la aparición de enfermedades cardiovasculares y resistencia a la insulina en el adulto. ${ }^{2.21}$ Es relevante en el momento de escoger el tipo de alimentación que se va a iniciar en el RNPT tener en cuenta sus antecedentes y patologías actuales para disminuir la incidencia de complicaciones relacionadas. La historia de alteraciones en el doppler de arterias placentarias con presencia de flujo diastólico reverso, se ha relacionado con mayor riesgo de desarrollar enterocolitis y problemas posteriores con la alimentación. ${ }^{22}$

\section{Requerimientos nutricionales en el RNPT}

Líquidos: su absorción en el RNPT se encuentra entre 96 y $200 \mathrm{ml} / \mathrm{k} /$ día lo que permite recibir estos aportes 
diarios por vía enteral. La recomendación actual es administrar entre 135 y $200 \mathrm{ml} / \mathrm{kdía}$ para los RNTP, considerando los valores cercanos al límite inferior en pacientes en riesgo de displasia broncopulmonar y ductus arterioso persistente. Si un $\mathrm{RN}$ recibe fórmula para prematuros estándar o leche materna con un volumen entre 150 y $180 \mathrm{ml} / \mathrm{k} /$ día es suficiente para cubrir las necesidades nutricionales. ${ }^{23} \mathrm{El}$ aporte de líquidos se inicia en forma progresiva en los primeros días de vida. Un posible esquema es ${ }^{22}$ el observado en la Tabla 1.

\begin{tabular}{|c|c|c|c|c|}
\hline \multicolumn{5}{|c|}{ Tabla I. Esquema de aporte de líquidos en RN } \\
prematuros \\
\hline \multirow{2}{*}{$\begin{array}{c}\text { Dia de } \\
\text { vida }\end{array}$} & \multicolumn{4}{|c|}{ ml/dia } \\
\cline { 2 - 5 }$<750 \mathbf{g}$ & $750-999 \mathrm{~g}$ & $1000-1.248 \mathrm{~g}$ & $>1.250 \mathbf{g}$ \\
\hline 1 & 12 & 12 & 12 & 24 \\
\hline 2 & 12 & 12 & 24 & 36 \\
\hline 3 & 24 & 24 & 36 & 48 \\
\hline 4 & 36 & 36 & 48 & 60 \\
\hline 5 & 48 & 48 & 60 & 72 \\
\hline 6 & 60 & 60 & 72 & 84 \\
\hline 7 & 72 & 72 & 84 & $96-108$ \\
\hline 8 & 84 & 84 & $96-108$ & $120-132$ \\
\hline 9 & 96 & $96-108$ & $120-132$ & $144-150$ \\
\hline 10 & $108-120$ & $120-132$ & $144-150$ & 150 \\
\hline 11 & $132-144$ & $144-150$ & 150 & 150 \\
\hline 12 & 150 & 150 & 150 & 150 \\
\hline
\end{tabular}

Energía: en condiciones normales los RN pueden retener entre 84 y $94 \%$ del aporte energético. El objetivo es alcanzar un crecimiento similar al intrauterino, con una distribución parecida de los nutrientes. El gasto de energía en el RN se distribuye así ${ }^{2}$ : tasa metabólica basal 40 a $50 \mathrm{kcal} / \mathrm{k} /$ día, mayor en los pequeños para la edad gestacional; actividad física 3,6 a 19 kcal/k/ día; termorregulación mínima por ambiente neutro; y crecimiento $10 \mathrm{kcal} / \mathrm{k} / \mathrm{d}$ ía. Como se puede ver, con un aporte de 90 a $120 \mathrm{kcal} / \mathrm{k} /$ día se pueden cubrir estas necesidades dejando un adecuado débito para acrecentar proteínas y grasas. En casos de aportes superiores a este límite, se presenta aumento de la ganancia de grasas con los riesgos consiguientes que implica, con aportes energéticos más bajos y además de no lograr un crecimiento adecuado se presenta un insuficiente uso de las proteínas (un gramo de proteína requiere $10 \mathrm{kcal}$ para su metabolismo). ${ }^{2} \mathrm{Un}$ rango adecuado de aporte calórico para estos niños es entre 110 y 135 $\mathrm{kcal} / \mathrm{k} / \mathrm{día} .{ }^{23}$ Siempre en el cálculo de las necesidades energéticas se deben tener en cuenta las enfermedades intercurrentes con las que cursa el paciente para el aumento correspondiente de las calorías. ${ }^{24}$

Proteínas: son muy importantes en el crecimiento de los RN ya que proveen un sustrato importante para la formación de los tejidos. Es fundamental tener equilibrio entre el aporte y el metabolismo, ya que si el primero es insuficiente se puede reflejar en un crecimiento inadecuado y cuando es excesivo hay acumulación de hidrogeniones y amonio. En los RNPT es importante tener en cuenta la incapacidad de sintetizar algunos aminoácidos esenciales como cisteína, taurina y glicina, que son necesarios en el desarrollo posterior. Con todo esto, la recomendación del aporte proteico para los $\mathrm{RN}$ con más de $1.000 \mathrm{~g}$ es de $3.5 \mathrm{a}$ $4 \mathrm{~g} / \mathrm{k} /$ día y en los que están por debajo de esta cifra entre 4 y 4,5 g/kdía ${ }^{23,25}$ iniciando en forma temprana, basados en una tasa obligada de pérdidas de $0,7 \mathrm{~g} / \mathrm{k} /$ día, una acreción proteica de $1,7 \mathrm{~g} / \mathrm{k} /$ día con una tasa de retención proteica de 80 a $90 \% .{ }^{26,27} \mathrm{La}$ indicación actual se basa en iniciar un aporte proteico desde el primer día de vida a razón de $1.5 \mathrm{~g} / \mathrm{k} /$ día con aumento progresivo de 0.5 a $1 \mathrm{~g} / \mathrm{k} /$ día. ${ }^{19,26}$

Hidratos de carbono: son importantes para prevenir el catabolismo, además de ser fuente rápida de energía y participar en el metabolismo de los aminoácidos y ácidos grasos no esenciales. En términos generales se recomienda una infusión entre 4 y 6 mg/k/min que permite asegurar un adecuado funcionamiento cerebral, eritrocitario, de médula renal y retina. El aporte de los carbohidratos debe oscilar entre 40 y $50 \%$ del aporte calórico total, sin superar $17 \mathrm{~g} / \mathrm{k} /$ día (en general 10 a $14 \mathrm{~g} / \mathrm{k} /$ día) para evitar los efectos deletéreos de la hiperglicemia y la sobrecarga de hidratos de carbono. ${ }^{2}$ 
Lipidos: son importante fuente de energía y necesarios para el aporte de los ácidos grasos esenciales (no hay producción endógena) linoleico y linolénico, relevantes en el aprendizaje y adecuado desarrollo visual de los RN. La recomendación actual es entre 40 y $55 \%$ del aporte calórico total $(4,4$ a 6 g/100kcal), con una relación de los ácidos linoleico/ linolénico entre 5:1 y 15:1 así como la adición de carnitina que favorece el transporte de ácidos grasos de cadena larga a través de la membrana mitocondrial. ${ }^{23}$

Calcio y fosforo: son los principales constituyentes del tejido óseo en el RN, su incorporación tiene especial importancia a partir de la semana 25 de edad gestacional con un pico entre las 36 y 38 . Por ello, podemos suponer un déficit importante de estos elementos en los RNTP. ${ }^{2}$ A pesar de conocer el déficit relativo de estos minerales en el RNPT, la evidencia no es contundente acerca de sus requerimientos y aportes que necesitan ni la manera de suplementarlos. Lo importante es hacer seguimiento del calcio, fósforo y fosfatasa alcalina séricos para evaluar la mineralización ósea y determinar sus aportes. ${ }^{2} \mathrm{El}$ consenso europeo recomienda entre 120 y $140 \mathrm{~m} / \mathrm{k} /$ día de calcio (se absorbe 50 a $60 \%$ ) y entre 60 y $90 \mathrm{mg} / \mathrm{k} /$ día de fósforo para disminuir riesgos de fractura y osteopenia, así como asegurar una adecuada mineralización. ${ }^{23}$

Hierro: en los RN prematuros es frecuente el desarrollo de ferropenia debido a que: 1) el hierro se acumula en el feto de manera principal en el tercer trimestre, 2) hasta el $80 \%$ de las embarazadas tienen déficit de hierro y 3) están expuestos a extracciones frecuentes de sangre. Esto hace que a menudo reciban transfusiones durante su estancia hospitalaria. La suplencia permite mejorar los depósitos de hierro normalizando las cifras de hemoglobina y reduciendo el desarrollo de anemia ferropénica a largo plazo. ${ }^{28}$ Parece obvio el beneficio de suplementar hierro pero es importante tener en cuenta que el exceso se relaciona con mayor estrés oxidativo que puede jugar papel en la retinopatía del prematuro y displasia broncopulmonar, por lo que se debe iniciar su administración a partir de la cuarta a sexta semanas de vida o de la última trasfusión recibida. Las necesidades diarias de hierro elemental son de
2 a $6 \mathrm{mg} / \mathrm{k} / \mathrm{día}$, siendo mayor en los RN de más bajo peso. ${ }^{13}$ Es importante dar el aporte de hierro lejos de las tomas para mejorar su absorción y evitar el efecto de disminuir la capacidad inmunológica de la leche materna. Las dosis de hierro elemental recomendadas por el SIBEN relacionadas con el peso ${ }^{2}$ se observan en la Tabla 2.

\begin{tabular}{|c|c|}
\hline \multicolumn{2}{|c|}{$\begin{array}{c}\text { Tabla 2. Requerimientos de } \\
\text { hierro elemental }\end{array}$} \\
\hline $\begin{array}{c}\text { Peso } \\
\text { (gramos) }\end{array}$ & $\begin{array}{c}\text { Dosis hierro elemental } \\
\text { (mg/k/día) }\end{array}$ \\
\hline$<1.000$ & $4-6$ \\
\hline $1.000-1.500$ & 3 \\
\hline $1.500-2.000$ & 2 \\
\hline
\end{tabular}

\section{Micronutrientes}

Las recomendaciones sobre los requerimientos de micronutrientes se ven en la Tabla $3{ }^{2}$

\begin{tabular}{|c|c|}
\hline \multicolumn{2}{|c|}{ Tabla 3. Recomendaciones de } \\
requerimientos de micronutrientes \\
\hline Elemento & Por kilo por día \\
\hline Cinc, mcg & 400 \\
\hline Cobre, mcg & 20 \\
\hline Selenio, mcg & $1,5-4,5$ \\
\hline Cromo, mcg & $0,05-0,3$ \\
\hline Manganeso, mcg & 1 \\
\hline Molibdeno, mcg & 0,25 \\
\hline Yodo, mcg & 1 \\
\hline Taurina, $\mathrm{mg}$ & $1,88-3,75$ \\
\hline Carnitina, mg & 2,9 \\
\hline Inositol, $\mathrm{mg}$ & 54 \\
\hline Colina, $\mathrm{mg}$ & $14,4-28$ \\
\hline
\end{tabular}


Vitaminas: se recomienda iniciar el aporte de suplementos vitamínicos $1 \mathrm{ml} /$ día a los $\mathrm{RN}$ que reciben lactancia materna exclusiva al tolerar el volumen de la alimentación completa, ya que tienen una osmolaridad elevada y pueden relacionarse con aumento de intolerancia al aporte enteral. Las recomendaciones de suplementos vitamínicos para $\mathrm{RN}$ prematuros que reciben nutrición enteral ${ }^{2}$ se enumeran en la Tabla 4.

Nutrición enteral mínima: desde el período fetal se usa el tracto gastrointestinal con ventajas en la maduración del mismo. Al final del tercer trimestre hay ingesta de líquido amniótico por el feto. ${ }^{12}$ La nutrición enteral mínima se define como el período de estimulación trófica en el que se administra durante varios días leche materna o fórmula entera o diluida en cantidades que no tienen consecuencia nutricional, mientras la nutrición parenteral es la fuente principal de nutrientes. En general se trata de volúmenes entre 10 y $24 \mathrm{ml} / \mathrm{k} /$ día, que permite mejorar el desarrollo del aparato gastrointestinal, con buen flujo sanguíneo al tracto, mejoría de la motilidad y grandes ventajas endocrinas, favoreciendo un mayor recambio celular a nivel de la pared intestinal y secreción de varias hormonas como gastrina, enteroglucagón y motilina entre otras ${ }^{29}$, que ayudan a la maduración del tubo digestivo sin aumentar el riesgo de enterocolitis necrotizante (gran temor que se tiene al iniciar la nutrición enteral en el RNPT) y logrando un aporte enteral pleno en menos días. ${ }^{2,30}$

Algunas de las ventajas mencionadas de la alimentación enteral trófica son que favorece la integridad

\begin{tabular}{|c|c|c|c|c|c|}
\hline & Bases & Recomendación & LM & $L M+F$ & F pre \\
\hline A & $\begin{array}{l}\text { Paso de la madre al feto en último trimestre a RNPT } \\
\text { con déficit. } \\
\text { Importante en crecimiento epitelial, desarrollo visual } \\
\text { de retina, sistema inmune. } \\
\text { Protector en displasia broncopulmonar. } \\
\text { Exceso: aumento de presión intracraneana, daño } \\
\text { hepático y hematológico. }\end{array}$ & 700 - I 500 UI/k/día & 337 & $\begin{array}{l}987- \\
1.760\end{array}$ & $330-1.641$ \\
\hline $\mathrm{D}$ & $\begin{array}{l}\text { Es una prohormona, requiere luz UV para } \\
\text { metabolizarse. } \\
\text { Juega papel importante en la homeostasia } \mathrm{Ca} / \mathrm{P} \text {. }\end{array}$ & 400 Ul/k/día & 20 & $93-320$ & $48-218$ \\
\hline$E$ & $\begin{array}{l}\text { Importante antioxidante, mantiene integridad de la } \\
\text { membrana celular. } \\
\text { Déficit asociado con anemia hemolítica y defecto de } \\
\text { fagocitosis. } \\
\text { No es claro en efecto protector contra DBP, ROP y } \\
\text { HIV. }\end{array}$ & $5-25$ Ul/k/día & Insuf & SUF & SUF \\
\hline K & $\begin{array}{l}\text { Su déficit se relaciona con enfermedad hemolítica del } \\
\text { RN. } \\
\text { Se debe aportar a RNPT alimentados con LM } \\
\text { exclusiva. }\end{array}$ & & & & \\
\hline C & $\begin{array}{l}\text { Importante en equilibrio oxidativo. Aporte suficiente } \\
\text { en fórmulas y LM fortificada. }\end{array}$ & $20-50 \mathrm{mg} / \mathrm{dí}$ & Insuf & Suf & Suf \\
\hline B9 & $\begin{array}{l}\text { Ácido fólico importante en la síntesis de ADN. } \\
\text { Requerimientos aumentados en RNPT. Aporte } \\
\text { incrementado a medida que se avanza LM. }\end{array}$ & $50-60 \mathrm{mcgr} / \mathrm{k} / \mathrm{d}$ & Suf & Suf & Suf \\
\hline
\end{tabular}

LM. leche materna; $L M+F$, leche materna fortificada; Fpre, fórmula para prematuros; DBP: displasia broncopulmonar; ROP, retinopatía del prematuro; HIV. hemorragia intraventricular; SUF, suficiente. 
funcional y estructural del aparato gastrointestinal, mejora el desarrollo del epitelio del intestino, la acción hormonal, la actividad de la lactasa, la absorción de alimentos y la mineralización ósea. ${ }^{2}$

En un metanálisis adelantado por la colaboración Cochrane $^{31}$ en el que se incluyeron nueve estudios con diferencias metodológicas importantes, se encontró que la alimentación enteral trófica mostró una reducción significativa en los días en alcanzar la enteral completa, en el tiempo en que se suspendió el aporte enteral por intolerancia y en la estancia hospitalaria, sin demostrar mayor riesgo de enterocolitis necrotizante en el grupo que inició temprano la alimentación enteral. Si bien al evaluar los riesgos de la enteral trófica, los estudios no demuestran diferencias significativas, los intervalos de confianza de estos estudios son amplios y no permiten sacar conclusiones contundentes, aunque la enteral trófica parece tener más beneficios que complicaciones. ${ }^{2}$ A pesar de esto falta unanimidad con respecto al momento de iniciar la alimentación enteral en los prematuros. ${ }^{32}$ Las únicas contraindicaciones absolutas para suministrar alimentación enteral trófica es la presencia de enterocolitis y alguna malformación de la vía enteral. Por lo demás, no existe evidencia clara sobre el uso de alimentación enteral trófica en presencia de sepsis, DAP en cierre, choque o uso de catéteres umbilicales. En estos casos la decisión de iniciar la vía enteral debe ser individualizada con las precauciones pertinentes. ${ }^{2}$ La recomendación actual es iniciar estimulación enteral trófica (10 a $20 \mathrm{ml} / \mathrm{k} /$ día) en las primeras horas o a más tardar en los tres primeros días de vida, con leche materna siempre que sea posible. Mantener este aporte por mínimo cuatro a siete días, antes de comenzar el incremento progresivo de los volúmenes de aporte enteral. ${ }^{10}$

Después de iniciada la alimentación enteral trófica la siguiente cuestión es definir la velocidad del aumento de la misma. La recomendación actual es incrementar 10 a $15 \mathrm{ml} / \mathrm{k} /$ día para los $\mathrm{RN}$ más enfermos o menores de 1.000 g y 15 a $20 \mathrm{ml} / \mathrm{k} /$ día para aquellos más estables y con peso entre 1.000 y $1.500 \mathrm{~g}^{2}$, aunque se han hecho estudios con aumentos de $30 \mathrm{ml} / \mathrm{k} /$ día mostrando adecuada tolerancia y mejorando la ganancia de peso y menos días de estancia hospitalaria, sin aumentar los riesgos en niños nacidos con 1.000 a 2.000 g. ${ }^{33-35}$ Evaluar la tolerancia al aporte enteral en los RNPT es difícil, si bien existen creencias sobre la relación de la presencia de residuo patológico (mayor de $2 \mathrm{ml} / \mathrm{k}$ o superior al 50\% de la toma previa) y distensión abdominal, no se ha demostrado por completo y se plantean nuevos estudios en este sentido. ${ }^{36,37}$ La tolerancia con la alimentación más frecuente y volúmenes bajos, cada dos horas, parece ser mejor que cada tres horas. ${ }^{38}$

Como norma el recién nacido requiere para la alimentación enteral de sonda naso u orogástrica (no hay diferencia entre una y otra ${ }^{39}$ ) y se debe realizar mediante aporte intermitente (cada tres horas) de manera lenta (por gavaje). ${ }^{40}$ No existe ventaja para recomendar la infusión continua u otra forma de alimentación. ${ }^{2,15}$ Se debe evitar el uso de sonda transpilórica pues no tiene ninguna ventaja y cursa con gran número de complicaciones. ${ }^{41}$ En algunos centros se ha utilizado la succión no nutritiva (con chupo) para mejorar la adaptación a la alimentación posterior, con mejoría en el tiempo de estancia hospitalaria y en la adaptación a la alimentación con biberón. ${ }^{42}$

No existe duda acerca de que el alimento ideal para los RNPT es la leche de la propia madre. ${ }^{36}$ Las ventajas son innumerables pero se destacan el neurodesarrollo y la disminución en la incidencia de procesos infecciosos, además de mejorar la tolerancia del aporte enteral completo y disminuir el riesgo de enterocolitis necrotizante. ${ }^{4,43-46}$ Un pequeño inconveniente radica en el bajo aporte calórico de la leche materna, ${ }^{47}$ pero esta situación se ha mejorado con la adición de fortificadores con lo que se logra mejorar el aporte nutricional al paciente. ${ }^{5,48}$ En general estos se adicionan de manera progresiva, (mayor riesgo de enterocolitis al aumentar la osmolaridad) cuando el $\mathrm{RN}$ reciba por lo menos 100 $\mathrm{ml} / \mathrm{k} /$ día iniciando a $22 \mathrm{kcal} / \mathrm{oz}$ y llegando a $24 \mathrm{kcal} /$ oz. ${ }^{2,46}$ Los fortificadores de leche materna se suspenden alrededor de las 36 a 38 semanas de edad gestacional corregida $(1.800-2.000 \mathrm{~g})$ siempre y cuando el niño amamante bien y no tenga algún déficit específico.

En caso de imposibilidad para administrar leche materna, se podría iniciar fórmula para prematuros 
fortificada (24 kcal/oz) preparada sin diluir pues la osmolaridad es igual a la fórmula de inicio. Se debe mantener hasta las 40 semanas de edad gestacional corregida o algunos días previos al alta hospitalaria. $^{2}$

Una vez dado de alta el RNPT presenta retos para continuar una adecuada ganancia de peso y evitar las complicaciones relacionadas con la pobre alimentación. El primer año de vida muestra un terreno importante para lograr recuperar las pérdidas acumuladas y mejorar el crecimiento y neurodesarrollo a largo plazo. Se conoce la mayor necesidad en especial de proteínas, calcio, fósforo y vitamina A. En general los pacientes que reciben lactancia materna aumentan su ingesta para compensar las necesidades calóricas, otra opción es continuar los fortificadores de leche materna al alta o aportar fórmula enriquecida extra a la alimentación materna, cualquiera de estas estrategias es válida y escoger una u otra se basa en el seguimiento del patrón de crecimiento y las preferencias individuales. En los niños alimentados con fórmula se han creado algunas con modificaciones (enriquecidas: 22 $\mathrm{kcal} / \mathrm{oz})^{+4}$ que han demostrado en los diferentes estudios mejorar la talla, el peso y el perímetro cefálico en el seguimiento a largo plazo..$^{2.50}$

\section{Conclusiones}

La alimentación en el RNPT juega un papel muy importante tanto en el control de patologías agudas y propias del período neonatal, así como en el pronóstico a largo plazo de los pacientes y se ha demostrado la relación de este tópico con el desarrollo de patologías en la edad adulta. La alimentación enteral temprana se presenta como una importante y segura alternativa para los prematuros y se debe desmitificar la creencia que se tiene con el desarrollo de enterocolitis. Es importante individualizar el manejo de cada paciente para evitar las complicaciones de la nutrición enteral temprana. Ya están bien establecidas las indicaciones de cada uno de los nutrientes que se deben aportar a los prematuros y se deben seguir estos lineamientos, teniendo en cuenta la patología particular de cada uno.

\section{Referencias}

1. Civardi E, Tzialla C, Garofoli F, Mazzucchelli I, Bollani L, Stronati M. Nutritional needs of premature infants. J Matern Fetal Neonatal Med. 2011 Oct:24 Suppl $1: 27-9$.

2. Rogido M, Golombek SG, Baquero H, Borbonet D, et al. Tercer consenso clinico SIBEN: nutricion del recien nacido enfermo; 2009 Sep.; Lima, Perù.

3. Knops NB, Sneeuw KC, Brand R, et al. Catch-up growth up to ten years of age in children born very preterm or with very low birth weight. BMC pediatrics. 2005:5:26.

4. Corpeleijn WE, Vermeulen MJ, van den Akker CH, van Goudoever JB. Feeding very-low-birth-weight infants: our aspirations versus the reality in practice. Ann Nutr Metab. 2011:58 Suppl 1:20-9.

5. Clark RH, Wagner CL, Merritt RJ, et al. Nutrition in the neonatal intensive care unit: how do we reduce the incidence of extrauterine growth restriction? J Perinatol. 2003 Jun;23(4):337-44.

6. Ehrenkranz RA, Dusick AM, Vohr BR, Wright LL, Wrage LA, Poole WK Growth in the neonatal intensive care unit influences neurodevelopmental and growth outcomes of extremely low birth weight infants. Pediatrics. 2006:117:1253-61.

7. Isaacs EB. Gadian DG, Sabatini S, et al. The effect of early human diet on cauda te volumes and IQ. Pediatr Res. 2008 Mar:63(3):308-14.

8. Brandt 1, Sticker EJ, Lentze MJ. Catch-up growth of head circumference of very low birth weight. small for gestational age preterm infants and mental development to adulthood. J Pediatr. 2003 May; 142(5):463-8

9. Wemhoner A. Ortner D, Tschirch E. Strasak A. Rudiger M. Nutrition of preterm infants in relation to bronchopulmonary dysplasia. BMC Pulm Med. 2011 Feb $3 ; 11: 7$

10. Smith JR. Early enteral feeding for the very low birth weight infant: the development and impact of a research-based guideline. Neonatal Netw. 2005 Jul-Aug; 24(4):9-19.

11. Embleton NE, Pang N, Cooke RJ. Postnatal malnutrition and growth retardation an inevitable consequence of current recommendations in preterm infants?. Pediatrics. 2001;107:270-3.

12. Adamkin DH. Nutrition Management of the Very Low-birthweight Infant. NeoReviews. 2006;7:e602-e7

13. Adamkin DH. Nutrition Management of the Very Low-birthweight Infant. NeoReviews. 2006; 7:e608-e14.

14. Hay WW, Jr. Strategies for feeding the preterm infant. Neonatology. 2008; $94: 245-54$

15. Wilson DC, Cairns P, Halliday HL, Reid M, McClure G, Dodge JA. Randomised controlled trial of an aggressive nutritional regimen in sick very low birthweight infants. Arch Dis Child Fetal Neonatal Ed. 1997 Jul;77(1):F4-11

16. Dincrstein A, Nieto RM, Solana CL, Perez GP, Otheguy LE, Larguia AM Early and aggressive nutritional strategy (parenteral and enteral) decreases postnatal growth failure in very low birth weight infants. J Perinatol. 2006 Jul;26(7):436-42.

17. De Curtis M, Rigo J. The nutrition of preterm infants. Early Hum Dev. 2012 Mar;88 Suppl 1:S5-7

18. Denne SC, Poindexter BB. Evidence supporting early nutritional support with parenteral amino acid infusion. Semin Perinatol. 2007 Apr;31(2):56-60.

19. Valentine CJ, Fernandez S, Rogers LK, et al. Early amino-acid administration improves preterm infant weight. J Perinatol. 2009 Jun;29(6):428-32.

20. Ehrenkranz RA. Early, aggressive nutritional management for very low birth weight infants: what is the evidence?. Semin Perinatol. 2007 Apr;31(2):48-55.

21. Leunissen RW, Oosterbeek P, Hol LK, Hellingman AA, Stijnen T, HokkenKoelega AC. Fat mass accumulation during childhood determines insulin sensitivity in early adulthood. J Clin Endocrinol Metab. 2008 Feb;93(2):445-51. 2008 Feb;93(2):445-51. 
22. Leaf A, Dorling J, Kempley S, McCormick K, Mannix P, Brocklehurst P. ADEPT - Abnormal Doppler Enteral Prescription Trial. BMC Pediatr. 2009 Oct 2;9: 63 .

23. Agostoni C, Buonocore G, Carnielli VP, et al. Enteral nutrient supply for preterm infants: commentary from the European Society of Paediatric Gastroenterology, Hepatology and Nutrition Committee on Nutrition. J Pediatr Gastroenterol Nutr. 2010 Jan;50(1):85-91

24. Hulzebos CV, Sauer PJ. Energy requirements. Semin Fetal Neonatal Med. 2007 Feb;12(1):2-10

25. Hay WW, Thureen P. Protein for preterm infants: how much is needed? How much isenough? How much is too much?. Pediatr Neonatol. 2010 Aug;51(4): 198 207

26. Thureen PJ, Melara D, Fennessey PV, Hay WW, Jr. Effect of low versus high intravenous amino acid intake on very low birth weight infants in the early neonatal period. Pediatr Res. 2003;53:24-32.

27. Denne SC. Protein and energy requirements in preterm infants. Semin Neonatol. 2001;6:377-82.

28. Mills RJ, Davies MW. Enteral iron supplementation in preterm and low birth weight infants. Cochrane Database Syst Rev. 2012 Mar 14;3: CD005095

29. Mishra S, Agarwal R, Jeevasankar M, Deorari AK, Paul VK. Minimal enteral nutrition. Indian J Pediatr. 2008 Mar;75(3):267-9

30. Karagianni P, Briana DD, Mitsiakos G, et al. Early versus delayed minimal enteral feeding and risk for necrotizing enterocolitis in preterm growth-restricted infants with abnormal antenatal Doppler results. Am J Perinatol. 2010 May; 27(5):367-73.

31. Tyson JE. Kennedy KA. Trophic feedings for parenterally fed infants. Cochrane Database Syst Rev. 2005 Jul 20;(3):CD000504

32. Klingenberg C, Embleton ND, Jacobs SE, O’Connell LA, Kuschel CA. Enteral feeding practices in very preterm infants: an international survey. Arch Dis Child Fetal Neonatal Ed. 2012 Jan;97(1):F56-61

33. Caple J, Armentrout D, Huseby V, et al. Randomized, controlled trial of slow versus rapid feeding volume advancement in preterm infants. Pediatrics. 2004;1 14:1597-600.

34. Morgan J, Young L, McGuire W. Slow advancement of enteral feed volumes to prevent necrotising enterocolitis in very low birth weight infants. Cochrane Database Syst Rev. 2011 Mar 16;(3):CD001241.

35. Krishnamurthy S, Gupta P, Debnath S, Gomber S. Slow versus rapid enteral feeding advancement in preterm newborn infants 1000-1499 g: a randomized controlled trial. Acta Paediatr. 2010 Jan;99(1):42-6.
36. Shulman RJ, Ou CN, Smith EO. Evaluation of potential factors predicting attainment of full gavage feedings in preterm infants. Neonatology. 2011;99:38-44.

37. Lucchini R, Bizzarri B, Giampietro S, De Curtis M. Feeding intolerance in preterm infants. How to understand the waming signs. J Matern Fetal Neonata Med. 2011 Oct; 24 Suppl 1:72-4.

38. DeMauro SB, Abbasi S, Lorch S. The impact of feeding interval on feeding outcomes in very low birth-weight infants. J Perinatol. 2011 Jul;31(7):481-6.

39. Hawes J, McEwan P, McGuire W. Nasal versus oral route for placing feeding tubes in preterm or low birth weight infants. Cochrane Database Syst Rev. 2004;(3):CD003952.

40. King C. What's new in enterally feeding the preterm infant? Arch Dis Child Fetal Neonatal Ed. 2010 Jul;95(4):F304-8

41. McGuire W, McEwan P. Transpyloric versus gastric tube feeding for preterm infants. Cochrane Database Syst Rev. 2007 Jul 18;(3):CD003487

42. Pinelli J, Symington A. Non-nutritive sucking for promoting physiologic stability and nutrition in preterm infants. Cochrane Database Syst Rev. 2005 Oct 19;(4):CD001071

43. Sisk PM, Lovelady CA, Gruber KJ, Dillard RG, O'Shea TM. Human milk con sumption and full enteral feeding among infants who weigh $</=1250$ grams Pediatrics. 2008;121:el 528-33.

44. Isaacson LJ. Steps to successfully breastfeed the premature infant. Neonatal Netw. 2006 Mar-Apr; 25(2):77-86.

45. McGuire W, Henderson G, Fowlie PW. Feeding the preterm infant. BMJ. 2004 Nov 20;329(7476): 1227-30

46. Diehl-Jones WL. Askin DF. Nutritional modulation of neonatal outcomes AACN Clin Issues. 2004 Jan-Mar;15(1):83-96

47. Arslanoglu S, Moro GE, Ziegler EE. Preterm infants fed fortified human milk receive less protein than they need. J Perinatol. 2009 Jul:29(7):489-92.

48. O'Connor DL, Khan S, Weishuhn K, et al. Growth and nutrient intakes of human milk-fed preterm infants provided with extra energy and nutrients after hospita discharge. Pediatrics. 2008;121:766-76.

49. Schanler RJ. Post-discharge nutrition for the preterm infant. Acta Paediatr Suppl 2005 Oct; 94(449):68-73

50. Cooke R. Nutrition of preterm infants after discharge. Ann Nutr Metab. 2011 158 Suppl 1:32-6 